\title{
A Pragmatic Analysis of the Discourse in Chu Long Persuades Empress Dowager Zhao
}

\author{
Yao $\mathrm{Xi}^{1{ }^{1 *}}$ \\ ${ }^{I}$ Dalian Neusoft University of Information, Dalian, Liaoning, China \\ axiyao@neusoft.edu.cn
}

\begin{abstract}
This paper attempts to analyze the discourse between Chu Long and Empress Dowager Zhao based on the pragmatic theories including the cooperative principle, the conversational implicature, the politeness principle, and the speech act theory, so that we can use an approximately scientific method to reinterpret the personality traits of the two main characters in Chu Long Persuades Empress Dowager Zhao, and deeply appreciate Chu Long's superb art of communication, which provides a good example for the superior-subordinate communication in the real life.
\end{abstract}

Keywords: cooperative principle, conversational implicature, politeness principle, speech act, Chu Long, Empress Dowager Zhao

\section{《触龙说赵太后》中人物对话的语用分析}

席瑶 ${ }^{1, a}$

${ }^{1}$ 大连东软信息学院, 大连, 辽宁, 中国

axiyao@neusoft.edu.cn

\section{摘要}

本文试从语用学理论中的会话合作原则、会话隐涵理论、礼貌原则以及言语行为对触龙与赵太后的对 话进行了分析, 从而使我们用近似科学的方法重新解读了《触龙说赵太后》中两位主要人物的性格特 征, 并深切领略了触龙高超的话语交际艺术, 为现实生活中上下级间的沟通交流提供一个很好的范例。 关键词: 会话合作原则、会话隐涵、礼貌原则、言语行为、触龙、赵太后

\section{1. 语用学理论}

\section{1. 会话合作原则}

话语交流是一种双边的或多边的语言行为, 交流双 方在开始一段对话时通常是有一个无须言明的前提的, 那就是本着合作的态度进行交流。合作可以避免交流者 无缘无故地迷惑对方、欺骗对方、藏匿重要信息, 从而 使双方在相互信任、理解的基础上将对话顺利地进行下 去。在这一合作态度的基础上, Grice $(1975,1978,1981)$ 提出了会话合作原则及其四条准则, Yule (1996) 对此 做出了如下概括:

"The cooperative principle: Make your conversational contribution such as is required, at the stage at which it occurs, by the accepted purpose or direction of the talk exchange in which you are engaged"[1]. 蓝纯 (2007) 将其 解释为 “当你参与一段会话时, 你所说的话应该符合当
时情景的要求, 并且在适当的时间、为双方所接受的目 的说出” [2]。

The maxims (会话合作准则): Quantity (适量准则); Quality (真实准则); Relation (相关准则); Manner (明 晰准则)。

\section{2. 会话隐涵}

尽管会话合作原则和四条准则是任何话语交流顺 利进行的出发点，但是并不是所有人在会话中都无时无 刻地遵循这些金科玉律。在这种情况下绝不是说会话者 就不再秉承合作的态度，而是为了传达某种特殊的交际 效果而故意、甚至公开地不遵循某一条或是某几条准 则, Grice 称这种现象为违反 (flouting)。同时也可能是 会话者巧妙地运用语言技巧, 故意不直接回答对方的问 题, 可能从表面看来是所问非所答, 但实际上是产生了 会话隐涵, 也就是说需要对话者从更深的层面解读对方 的意图。会话隐涵不是产生于孤立的句子, 而是依赖于 
话语的具体语境以及说话人和听话人之间共享的背景 知识。根据会话隐涵的推导过程对上下文的依赖程度， 可以将会话隐涵分为两类: 分别是会话隐涵和特殊会话 隐涵。前者指的是无须借助会话所发生的语境的特殊背 景知识就可推导出来的言外之意, 后者指的是必须借助 这些特殊的背景知识方可推导出来的言外之意。

\section{3. 礼貌原则}

礼貌原则的存在是为了维持社会的稳定与和谐, 维 护人与人之间的友善关系。因为有了这种和谐与友善, 我们才可以放心地假定进入任何一段对话的双方都是 抱着合作态度的, 所以会话合作原则与礼貌原则是密不 可分的。

Lakoff（Lakoff，1973；蓝纯，2007）是最早尝试 以 Grice 的会话合作原则来解释礼貌现象的语言学家之 一。她提出了两条语用能力原则：1）说清晰的话；2） 说礼貌的话。关于礼貌, Lakoff 又进一步提出了三条具 体的规则, 即: 不要强加于人; 提供选择; 让对方感觉 舒服。Leech (1983) 列出了比 Lakoff 更详尽的六条礼 貌准则: Tact Maxim (策略准则); Generosity Maxim (慷慨准则); Approbation Maxim (赞扬准则); Modesty Maxim (谦虚准则); Agreement Maxim (赞同准则); Sympathy Maxim（同情准则） ${ }^{[3]}$ 。

Lakoff 和 Leech 都是在观察英国社会阶层的基础上 总结出的礼貌准则, 而由于各个国家语言与文化的差 异, 这些准则并不是百分之百地适用于其他国家的各社 会阶层。于是顾曰国（顾曰国, 1992; 蓝纯, 2007) 针 对汉语的礼貌现象, 对比 Leech 的礼貌准则, 总结出了 五条适用于汉语的礼貌准则, 即: 贬已尊人准则、称呼 准则、文雅准则、求同准则和德、言、行准则。

\section{4. 言语行为}

言语行为理论最早是由英国哲学家 Austin 提出的, 他认为我们用语言表达时, 实际上是实施了言语行为, 即 “以言行事”。后来经过美国语言学家 Searle 的发展, 这一理论得到广泛地推广。为了描述言语行为所包含的 不同层面, Austin (1962) 提出了发话行为, 行事行为 和取效行为 ${ }^{[4]}$ 。他认为在任何情况下, 实施一个言语行 为意味着同时实施三个相关的行为。第一种是普通意 义, 是指我们说话的时候, 要移动发音器官, 发出按照 一定方式组织起来, 并被赋予了一定意义的声音。然而 事实上, 我们在说话时, 不只是说出一些具有一定意义 的语言单位, 而且说明我们的说话目的, 也就是 Austin 说的具有一定的语力。语力相当于说话者意义, 语境意 义, 或附加意义。而说话可以看成做事的第三种意义, 涉及话语对听话人产生的效果。通过告诉听话人某事, 说话人可以改变听话人对某件事的观点, 或者误导他, 让他惊奇, 诱导他做某事等等, 也就是 Austin 所说的取 效行为。

\section{2. 《触龙说赵太后》中人物对话的语用分析}

左师触龙说希望谒见太后。太后听后以为触龙与其 他大臣一样也是来当说客的, 于是先从行动上表现出了 不合作与不礼貌, 她怒容满面地等待触龙进谏。触龙进 来后慢步走向太后, 到了跟前请罪说: “老臣脚有病, 已经丧失了快跑的能力, 好久没能来谒见了, 私下里原 谅自己, 可是怕太后玉体偶有欠安, 所以很想来看看太 后。”在封建社会里, 等级制度森严, 所以君臣之间说 话时一定要把握好尺度与分寸, 社会地位的不同便决定 了所用称呼的不同以及说话人与听话人的亲疏关系。在 这一点上触龙可以说是拿捏地非常到位, 合理地遵循了 会话中的礼貌准则。因为中国古代很讲究用谦称和尊 称, 大臣们在君主面前一定要将自己称为 “臣” 或者 “微 臣”。而在这里触龙称自己为 “老臣” 首先说明他遵循 了 Leech (1983) 礼貌准则中的谦虚准则, 也可以说是 遵循了顾曰国（1992）礼貌准则中的贬已尊人准则, 即 在指谓自己或与自己相关的事物时要 “贬”、要 “谦”; 在指谓听者或听者有关联的事物时, 要 “抬”, 要 “尊”。 而且触龙称自己为 “老臣” 意为表现自己是元老级臣子, 较 “微臣” 和 “臣” 更拉近了与太后之间的距离。并且 他将太后的身体称为 “玉体”, 突显了太后的尊贵, 表 现了对太后的尊重。太后说: “我老妇行动全靠手推 车。”太后的这句话还是遵循了Grice 的会话合作原则, 但与此同时也包含了一定地会话隐涵。当触龙询问太后 的身体情况时，太后并没有直接回答身体的好坏，而是 用 “行动全靠手推车” 来说明自己行动的不方便，身体 欠佳。触龙于是又说: “每天的饮食该不会减少吧?” 太后说: “就靠喝点粥罢了。”太后同样运用了会话隐 涵来回答触龙的话, 她没有直接说饮食减少或没有, 而 是用只喝点粥说明饮食减少了。触龙见太后的饮食不是 很好便表现出了对太后的关切, 即遵循 Leech（1983） 礼貌准则中的同情准则。触龙很想给太后一些增加饮食 的建议，但由于触龙与太后间的等级地位，他不可能以 同等人的身份对太后指手画脚，建议太后如何去做，他 只能间接地以自身为例加以分析说明。触龙说: “老臣 现在胃口很不好, 就自己坚持着步行, 每天走三四里, 稍为增进一点食欲, 对身体也能有所调剂。”没想到太 后听后非但没有接受触龙的建议, 而且还表现得很不合 作、不礼貌。太后说: “我老妇可做不到。”即便说太 后一再地以一种盛气地姿态与触龙对话, 但由于触龙巧 妙地把握交际对象的特点, 有意识地将自己显示出老 态，并且从老年人普遍关注的养生问题谈起，使太后接 话 “恃劷而行” “恃粥耳” “老妇不能”, 很符合语言背 景这一制约话语交际要素的要求。在同一年龄谈同一话 题, 自然不会 “话不投机半句多”, 而是 “酒逢知已千 杯少”。由于把握好了交际目的和交际对象及语言背景 等要素, 原来怒气冲冲的赵太后此时自然 “色少解”, 脸色稍微缓和了, 使得触龙的话语交际能够顺利进行。

当然, 这样的寒暄、问候, 以及大谈 “养生之道” 显然不是触龙的真正的交际目的。如何达到真正的交际 目的 “质长安君于齐” 呢? 触龙的办法是运用制约话语 交际的另一因素一一话语场景。不同的话语场景决定了 
不同的话语交际。触龙偷偷地改变了话语交际的场景, 话题转为为儿子求职。他说: “老臣的劣子舒祺, 年纪 最小, 不成才。臣子老了, 偏偏爱怜他。希望能派他到 侍卫队里凑个数, 来保卫王宫。所以冒着死罪来禀告 您。”无论话语交际的场景、话题如何转变, 触龙仍旧 秉承着会话合作与礼貌准则, 首先在称呼上将自己的儿 子谦称为 “贱息” , 并且贬低其 “不成才”, 只希望能 到侍卫队充个数, 处处遵循着谦虚准则。末了他还不忘 自贬一句 “没死以闻”, 更是把谦虚准则奉行到底。触 龙的这一席话使太后听后产生一种错觉, 以为触龙是为 儿子 “走后门” 的, 不是为长安君的, 所以表现得很是 合作，一下子便同意了触龙的请求，而且为了维持双方 会话的连贯性, 还询问了一句 “年几何矣? ” 触龙回答 说: “十五岁了。虽然还小, 希望在老臣没死的时候先 拜托给太后。”说到这句时触龙更是将自己的生老病死 比喻成 “填沟壑”, 而这在古代通常是指贱民奴隶的死, 所以从一点可以看出触龙在赵太后面前时刻表现出自 己的卑微身份, 紧遵谦虚准则。触龙这样说一方面可以 突显赵太后高高在上的君主地位，另一方面又让赵太后 感觉这个年老的大臣跟自己的想法相同, 都痛爱小儿 子, 不但应该彼此关切, 而且志同道合了。于是接下来 的对话太后表现得越来越合作, 开始主动地向触龙询 问, 以确保对话的顺利进行。太后说: “做父亲的也爱 怜他的小儿子吗? ”触龙回答说：“比做母亲的更 爱。”触龙面对太后的提问并没有正面回答而是做出了 一定地会话隐涵, 意思是想告诉太后天下的父母都一 样, 无不疼爱自己的子女的, 而怜爱也绝不是母亲 (尤 其是太后）的专利, 父亲也一样疼爱儿子。太后笑道:

“妇道人家特别喜爱小儿子。”这样交际话题就自然而 然地转到 “子女” 问题上来了, 慢慢地再谈到长安君, 自然就水到渠成了。

触龙紧接着说: “老臣个人的看法, 老太后爱女儿 燕后, 要胜过长安君。” 触龙并没有直接说太后之爱, 而是将太后称为 “媼”, 或多或少还是违背了礼貌准则 的。因为 “媪” 是对古代妇女的通称, 而赵太后作为一 国君主, 不应该与其她妇女相提并论, 所以一直将礼貌 准则运用得恰到好处的触龙在这一点上有些欠妥当。但 或许是因为触龙与太后找到了共同话题的缘故, 这并没 有影响他跟太后之间的对话, 相反拉近他和太后的距 离, 太后不再那么盛气凌人, 高高在上, 而也尊称触龙 为 “君”。太后说: “您错了, 比不上对长安君爱得深。” 左师公再说: “父母爱子女, 就要为他们考虑得深远一 点。老太后送燕后出嫁的时候, 抱着她的脚为她哭泣, 是想到可怜她要远去, 也是够伤心的了。送走以后, 并 不是不想念她, 每逢祭祀一定为她祈祷, 祈祷说: ‘一 定别让她回来啊! , 难道不是从长远考虑, 希望她有了 子孙可以代代相继在燕国为王吗? ”触龙抓住太后爱 子情切, 将心比心, 充满了智慧和情感。在谈到燕后时, 说到太后 “持其踵, 为之泣”, 祈祷时 “必勿使返”, 条 分缕析, 感情真挚。指明太后对女儿的前途是有长远的 考虑的, 从对燕后的态度来看, 太后是深明大义的。太 后听后虽只以一个 “然” 字相回应, 但短短的一字正体 现了赵太后秉承了会话合作的原则, 为确保双方对话的
持续进行，在该接话的地方恰到好处地接上一句。最后， 触龙话语一转从 “三世以前” “赵之为赵”, 谈到 “虽曰 爱之, 其实害之” “一旦山陵崩, 长安君何以自托于赵”, 欲擒故纵, 击中对方要害。赵太后当然也不是不懂政治 的女人, 因此就痛快地答应了。在这样一个特定的话语 场景下，触龙实现了自己的交际目的，说服赵太后 “质 长安君于齐”。而在最后的对话中, 触龙还巧妙地对赵 太后实施了言语行为。左师公说: “这是他们近的灾祸 及于自身, 远的及于他们的子孙。难道是君王的子孙就 一定不好吗? 地位高人一等却没什么功绩, 俸禄特别优 厚却未尝有所操劳，而金玉珠宝却拥有很多。现在老太 后给长安君以高位, 把富裕肥沃的地方封给他, 又赐予 他大量珍宝, 却不曾想到目前使他对国家做出功绩。有 朝一日太后百年了, 长安君在赵国凭什么使自己安身立 足呢? 老臣认为老太后为长安君考虑得太短浅了, 所以 我以为你爱他不如爱燕后。”从表面看来触龙只是单纯 地谈论君王将相子女衣食无忧的一个现状, 而到最后也 只字未提送长安君到齐国当人质的事宜, 还只是在赵太 后对燕后与长安君的疼爱方面做对比, 但是他对长安君 未来前途的分析实际上是想劝说太后应该以大局为重, 只有先保全国家，才会有长安君美好的将来。当然最终 太后的一句 “诺。恣君之所使之” 使触龙的话取得了他 预期的效果, 也就完成了言语行为中的取效行为。

\section{3. 总结}

会话交流是一种双边或多边的语言行为, 若想保证 交流的顺利进行，会话双方是要共同遵循一些交流原则 的, 如会话合作原则、礼貌原则等。《触龙说赵太后》 就语用学理论在现实生活中的运用为我们提供了一个 很好的范例, 尤其体现了古人在上下级沟通方面的卓越 智慧，非常值得当今职场上的人们反思借鉴。

从《触龙说赵太后》的案例中我们会发现, 无论是 古代还是现代要实现有效的语用沟通都不是一件简单 的事情。若想保证沟通的顺利进行，首先会话的双方应 该保持合作交流的态度，然后再基于双方的身份、地位， 以及远近亲疏关系来遵循不同的礼貌原则。因为会话沟 通本身也是交谈双方的一次博弯，所以交谈者要不断揣 摩对方的会话涵义, 要去分析是否包含会话隐涵。同时, 在开始会话前要做足准备, 尤其对于上下级沟通而言, 下级如果想赢得谏言的机会, 那就必须要对上级领导的 脾气秉性和背景特点做好了解。所谓 “知已知彼、百战 不殁”, 只有建立在对双方足够了解的基础上，才能巧 用会话策略, 做到推心置腹、推己及人。正如李海涛 （2019）所说, “沟通者不仅要开动脑筋, 用智慧找寻 有效沟通的策略和方法, 确定解决实际问题的路径与方 案, 还要注意做到以情动人, 懂得换位思考、将心比心 的道理。只有真正走进对方内心，才能充分发挥沟通效 用, 也才是成功的沟通” [5]。 


\section{REFERENCES}

[1] George Yule (1996). Pragmatics[M]. Shanghai: Shanghai Foreign Language Education Press.

[2] Lan Chun (2007). A Pragmatic Approach to A Dream of Red Mansions[M]. Beijing: Foreign Language Teaching and Research Press.

[3] Geoffrey N. Leech (1983). Principles of Pragmatics[M]. Longman, London and New York.

[4] Austin, J. L. (1962). How to Do Things with Words[M]. Oxford: Oxford University Press.

[5] Li Haitao (2019). The Implication of Chu Long Persuades Empress Dowager Zhao for the Superior-Subordinate Communication $[\mathrm{J}]$. Leadership Science, 13:103-105. 